\title{
Asymétrie d'Information et les Trois Marchés d'Assurance
}

\author{
par Jean-Jacques Laffont *
}

La proposition stimulante de Monsieur le Professeur Borch est que les trois classes d'assurance qu'il envisage posent des problèmes différents à la théorie économique. Je vais discuter cette proposition sur un point précis : est-ce que les asymétries d'information entre assureurs et assurés doivent être traitées différemment dans les différentes classes d'assurance?

Dans une première partie je discute de façon informelle quelques différences entre les trois classes et je définis une problématique qui permettrait d'apporter une réponse à la question ci-dessus. Dans une deuxième partie je montre comment les progrès récents de la théorie des incitations permettent de poser clairement le problème de la construction d'une tarification incitative, qui est une des politiques à envisager face à l'information imparfaite.

Dans l'assurance vie l'objet d'incertitude est la date de décès du sujet ; l'information asymétrique sur cet événement concerne la santé du sujet.

Dans l'assurance affaires, il y a ici plusieurs risques de natures très différentes; les risques techniques associés au processus de production, tels les risques climatologiques pour l'agriculture ; les risques financiers associés à l'absence de marchés futurs et donc aux prix aléatoires ; les risques associés à la qualité de la gestion qui ont la nature particulière de pouvoir être fortement influencés par les agents eux-mêmes d'une façon non observable par l'assureur (problème de risque moral).

En présence d'un système de marchés des valeurs qui déjà conduit à un partage satisfaisant des risques, il n'y a pas de place pour l'assurance. Les actionnaires ont l'incitation à connaître au mieux les risques de l'entreprise. Toutefois, s'ils ont l'impression de ne pas pouvoir individuellement bien connaître certains risques, ils peuvent souhaiter qu'une assurance qui joue un peu le rôle d'une coalition d'actionnaires obtienne l'information et élimine certains risques qu'ils ont des difficultés à appréhender.

* Professeur à l'Université des Sciences Sociales de Toulouse; Directeur d'Etudes à l'Ecole des Hautes Etudes en Sciences Sociales, Paris. 
Lorsque la propriété de l'entreprise est peu disséminée ou pas du tout, il est souhaitable que les entreprises puissent s'assurer.

Dans l'assurance ménages, l'agent fait face à plusieurs types de risques et il ne peut pas partager ses risques autrement que par l'assurance. Souvent il peut influencer son risque par ses actions non observables (assurance auto) et souvent il connaît beaucoup mieux son risque que l'assureur (assurance incendie, vol), etc.

Quelles sont les solutions? Je vois trois types d'instruments.

1. Surmonter l'obstacle informationnel et connaître au mieux le risque ainsi que l'action de l'agent qui influence le risque, ceci est coûteux : examen médical pour l'assurance vie.

2. Utiliser au mieux l'information passée ; ceci peut conduire à des contrats complexes : bonus, malus.

3. Construire des contrats qui conduisent à une discrimination en faisant croître le prix avec la quantité d'assurance.

La problématique générale consiste à déterminer la combinaison optimale de ces instruments pour chaque type d'assurance.

Le ratio coûts de transaction sur montants des dommages, la forme spécifique de la fonction de coût d'acquisition d'information seront des paramètres essentiels pour déterminer la forme de la solution optimale.

La solution sera donc différente suivant les différentes classes d'assurance envisagées par Monsieur Borch justifiant les distinctions qu'il envisage mais cependant la démarche me semble commune dans les trois cas. Pour l'assurance vie on s'attend à ce que l'instrument 1 soit dominant mais pas nécessairement le seul utilisé. Pour les assurances ménages une combinaison des instruments 2 et 3 semble souhaitable.

Dans mon commentaire cette problématique ne peut rester que sous la forme de programme de travail.

Je souhaite seulement vous montrer comment les progrès récents de l'économie des incitations permettent de poser clairement le problème de la construction de contrats incitatifs, c'est-à-dire l'instrument 3 dont j'ai parlé plus haut.

Considérons le problème suivant : la population est composée d'un grand nombre d'agents qui ont une probabilité d'accident $\pi, \pi \in[\underline{\pi}, \bar{\pi}]$. On suppose que pour chaque valeur de $\pi$ il y a un grand nombre d'agents.

Le problème est de trouver des tarifs où la prime dépend de la quantité d'assurance achetée qui permettent de discriminer entre les agents.

Le principe de révélation ${ }^{1}$ nous dit qu'un tarif $p(z)$ est équivalent à un mécanisme de révélation dans lequel on demande à l'agent la révélation de son risque d'accident. C'est pourquoi je formule le problème en termes de recherche de mécanismes de révélation.

1 Voir Green et Laffont [1979]. 
Considérons un modèle à deux périodes dans lequel la fonction d'utilité d'un agent type s'écrit :

$$
x_{0}+\pi u\left(x_{1}\right)+(1-\pi) u\left(x_{2}\right),
$$

où $x_{0}$ est sa consommation dans la période $0, x_{1}$ sa consommation dans la période 1 , s'il a un accident, $x_{2}$ sa consommation dans la période 2 , s'il n'a pas un accident.

A la période 0 l'agent a une ressource $R_{0}$ et à la période 1 une ressource $R$ sauf s'il a un accident avec probabilité $\pi$; dans ce dernier cas il n'a aucune ressource.

L'agent va s'assurer à la période 0 en payant une prime $p$ pour recevoir une indemnité $z$ lorsqu'il a un accident dans la période 1 .

Un mécanisme de révélation est un couple de fonction $p(\pi), z(\pi)$ qui spécifient le montant de la prime et de l'indemnité pour chaque annonce de probabilité d'accident.

Un mécanisme de révélation est réalisable si

$$
p(\pi)=\pi z(\pi) \quad \text { pour tout } \pi \text { dans }[\underline{\pi}, \bar{\pi}],
$$

c'est-à-dire si l'assurance est en équilibre budgétaire pour chaque type de risque.

Un mécanisme de révélation conduit l'agent à dire la vérité si $\pi=\hat{\pi}$ est sa meilleure réponse dans le programme de maximisation :

$$
\begin{aligned}
& \operatorname{Max} x_{0}+\hat{\lambda} u\left(x_{1}\right)+(1-\hat{\pi}) u\left(x_{2}\right) \\
& \left(x_{0}, x_{1}, x_{2}, \pi\right) \\
& x_{0}=R_{0}-p(\pi) \\
& x_{1}=z(\pi) \\
& x_{2}=R \quad x_{0} \geqslant 0 \quad x_{1} \geqslant 0 \quad x_{2} \geqslant 0
\end{aligned}
$$

ou

$$
\operatorname{Max}\left[R_{0}-p(\pi)+\hat{\pi} u(z(\pi))+(1-\hat{\pi}) u(R)\right]
$$

$(\pi)$

d'où la condition nécessaire du $1^{\text {er }}$ ordre

$$
\frac{d p}{d \pi}(\pi)=\pi \frac{d u}{d x_{1}} \cdot \frac{d z}{d \pi}
$$

qui doit être vraie pour tout $\pi$ si on veut que le mécanisme extraie la vérité dans toutes les circonstances. 
La condition de réalisabilité

(2)

$$
p(\pi)=\pi z(\pi)
$$

implique

(3)

$$
\frac{d p}{d \pi}=z(\pi)+\pi \frac{d z}{d \pi}
$$

En utilisant (2) et (3), (1) peut s'écrire :

(4)

$$
\pi \frac{d z}{d \pi}\left[\frac{d u}{d x_{1}}(z)-1\right] \equiv z
$$

Pour pouvoir intégrer explicitement l'équation différentielle (4), prenons $u(\bullet)=\log (\bullet)$

(4) devient :

$$
\begin{aligned}
& \frac{(1-z) d z}{z^{2}}=\frac{d \pi}{\pi} \\
& \text { ou } \quad \pi=e^{K-\frac{1}{z}-\log z},
\end{aligned}
$$

d'où la classe de contrats non linéaires paramétrés par $K$

$$
p(z)=z e^{K-\frac{1}{z}-\log z}
$$

Il faut vérifier si les conditions du $2^{e}$ ordre sont réunies. La condition du $2^{e}$ ordre locale s'écrit :

$$
\begin{aligned}
& \frac{d u}{d x_{1}} \frac{d z}{d \pi} \geqslant 0 \\
& \text { d'où } \quad \frac{d z}{d \pi} \geqslant 0 .
\end{aligned}
$$


Cette condition nécessaire est aussi suffisante ; en effet

$$
\begin{aligned}
& \left\{R_{0}-p(\hat{\pi})+\hat{\pi} u(z(\hat{\pi}))+(1-\hat{\pi}) u(R)\right\}-\left\{R_{0}-p(\pi)+\hat{\pi} u(z(\pi))+(1-\hat{\pi}) u(R)\right\} \\
& =p(\pi)-p(\hat{\pi})+\hat{\pi}[u(z(\hat{\pi}))-u(z(\pi))] \\
& =(\pi-\hat{\lambda}) u(z(\hat{\pi}))+\int_{\pi}^{\pi} u(z(t)) d t \geqslant 0
\end{aligned}
$$

d'après la monotonicité faible de $u(z(\bullet))$.

La solution obtenue en (4) doit vérifier $\frac{d z}{d \pi} \geqslant 0$ d'où $z \leqslant 1$ ou $K \geqslant 1+\log \pi$.

Comme les fonctions d'utilité sont décroissantes en $K$, le meilleur contrat imposé par la concurrence, correspond à $K=1+\log \pi$, c'est-à-dire en revenant à la formulation de prime non linéaire :

$$
p(z)=z e^{1+\log \pi-\frac{1}{z}-\log z}
$$

qui est à comparer avec le contrat en information parfaite qui est :

$$
p(z)=z \pi \quad \text { pour tout } \pi \in[\underline{\pi}, \bar{\pi}]
$$

Le prix unitaire $\pi e^{1-\frac{1}{z}-\log z}$ est croissant avec la quantité d'assurance achetée à un taux décroissant. Les agents qui ont le plus mauvais risque $\bar{\pi}$, s'assurent comme dans le contrat à information parfaite $(z=1)$, les autres s'assurent moins. On retrouve ici un résultat classique; ce sont les bons risques qui sont pénalisés par l'asymétrie d'information.

\section{REFERENCE}

GREEN, J., et LAFFONT, J. J. [1979] : Incentives in Public Decision Making, North Holland, Amsterdam. 